\title{
Drugging the undruggable: targeting challenging E3 ligases for personalized medicine
}

\author{
"A balanced risk/gain assessment should be considered before starting a drug \\ discovery project aiming to target E3 ligases and, crucially, the amount of knowledge \\ for a given E3 ligase can foretell the chances of success."
}

First draft submitted: 17 January 2017; Accepted for publication: 26 January 2017; Published online: 6 March 2017

Keywords: E3 ligases $\bullet$ personalized medicine $\bullet$ protein-protein interactions $\bullet$ undruggable targets

Drug discovery is one of the most challenging, slow and expensive scientific ventures, where failure is the norm rather than the exception. Although the number of new drugs approved in the last years hits at a possible change of trend and justifies cautious optimism [1], phase II attrition is still far too high, and the overall return on investment for pharmaceutical research needs to improve. There is no doubt that new strategies to treat patients and to develop innovative drug candidates that are more likely to display efficacy and safety in the clinic are needed. In parallel, we are entering into a new revolution in therapeutics, that of personalized medicine. Nowadays, it is still unknown if personalized medicine will reduce attrition of clinical trials, but from a drug discovery point of view, personalized medicine opens new challenges and opportunities, as it can be deduced by rephrasing the Nobel Prize Aaron Ciechanover: "Until recently, we grouped breast cancer as one disease. But it turned out that it is a large group of diseases that are very different from one another." This perspective raises novel questions: Have we identified the targets to treat these individual diseases? Do we have drugs for all of these targets?

In recent years, the development of selective kinase inhibitors has emerged as an important class of anticancer agents [2]. These discoveries have prompted efforts to stratify patients before treatment based on specific genomic biomarkers with the final goal of optimizing clinical outcomes through personalized treatments. But, beyond kinases, could other families of targets behave similarly? Could selective kinase therapies provide a framework for the development of other target-class selective therapies?

The ubiquitin-ligase family is an ideal candidate to search for alternative therapeutic strategies. The Ubiquitin-Proteasome System (UPS) is a highly regulated mechanism that controls intracellular protein degradation and turnover [3]. Protein ubiquitination occurs through a cascade of enzymatic reactions, involving an E1 ubiquitin-activating enzyme, an E2 ubiquitin-conjugating enzyme and, finally, an E3 ubiquitin-ligase enzyme complex. Repeated iterations of this ubiquitination process, result in long chains of ubiquitin repeats on a given substrate and posterior degradation by the proteasome. Interestingly, E3 ubiquitin ligases (of which $>700$ are known in humans) confer substrate specificity to the protein ubiquitination pathway, making this unconventional enzyme class very attractive targets for specific and less toxic therapeutic intervention, reducing the promiscuity that can be related with other UPS components (i.e., proteasome inhibitors). Being a clear opportunity, the development of small molecules against E3 ligases has been rewarded with very limited success, leading to the idea that they are
Carles Galdeano

Facultat de Farmàcia i Ciències de I'Alimentació, Institut de Biomedicina (IBUB), Universitat de Barcelona. Av. Joan XXIII 27-31, 08028 Barcelona, Spain Tel.: +34 934029002; cgaldeano@ub.edu 
undruggable targets [4]. Three compounds (thalidomide, pomalidomide and lenalidomide) targeting the E3 ligase cereblon have been approved for the treatment of multiple myeloma or mantle cell lymphoma [5], and only a handful of compounds targeting the XIAP E3 ligase (AEG 35156), IAP (LCL161, Biripant, GDC0152, AT-406, AEG48826) and the MDM2 E3 ligase (RG7112, JNJ-26854165) have entered clinical trials. Additional research efforts have focused on targeting other E3 ligases, including Skp2, $\beta$ TrCP, Fbox3, VHL and Parkin. However, there is a significant difference between the number of $\mathrm{E} 3$ ligases and the number of drugs in clinical trials or approved. Overall, it can be considered that the field is still in its infancy.

"Several computational tools have been developed in the last few years to identify new binding sites and predict their druggability."

Why does the development of novel E3 ligase molecules remain a relatively untapped source by pharmaceutical industry? Although we are experiencing an exponential growth in knowledge about biological components of the E3 ligases (structure, function, localization and regulation) and how they relate to other components (physical interaction, post-transductional modifications and regulation of gene expression) [6], the general biology and chemistry of the E3 ligases is complex and not well understood. Moreover, linking individual E3 ligases to their substrates is an essential previous step. This relies on either a functional connection or a physical association between proteins; however, biochemical screens have proven not to be effective to identify E3 substrates, as the binding between E3 ligases and substrates is often intrinsically weak. An expansion of the biological and mechanistic information of E3 ligases, their substrates, and finally how they link to diseases will be crucial to increase the success of future drug discovery entrepreneurships.

\section{Drug discovery approaches to target E3 ligases}

Modulating the activities of E3 ligases requires targeting protein-protein interactions (PPIs). PPIs expose large and flat contact surfaces, which make daunting the perspective of targeting them with drug-like compounds [7]. Several general approaches have been applied to target PPIs in drug discovery programs that can also be applied to target the PPIs related to E3 ligases. Structure-based drug discovery is the obvious approach to follow, even more when tridimensional structures of substrate degrons in complex with E3 ligases or E3 ligases with other UPS components are available. Disruption of these PPIs with small molecules should provide a biologi- cal response through inhibition (or activation!) of the E3 ligase function or the assembly of the multisubunit protein complex. Noteworthy, often the loss of E3 protein activity leads to disease and a small molecule which is able to restore that function will be needed [8]. In the case of substrate degrons in complex with E3 ligases, a successful strategy consists of starting from the post-translational modified amino acids of the degron and to grow the molecule rationally, often with the help of computational tools (e.g., de novo design), to maximize the interactions with the E3 ligase surface [9,10]. However, it is not always possible to obtain a high-quality structure well suited for rational design. In the field of E3 ligases, this situation is even more complicated due to the need to obtain structural data of multisubunit protein complexes. In the last years, cryo-EM has emerged as a promising new technique to obtain structural data from challenging proteins [11], but it is unclear whether this technique will provide the fine atomic detail needed for structure-based drug discovery. Moreover, the availability of an E3 structure does not guarantee the success of a drug discovery research program in this field. As an example, the x-ray structure of Fbw7 was deposited in the Protein Data Bank (PDB) in 2007 [12]. Given the key role of Fbw7 in tumorogenesis, one could anticipate that small molecules directly targeting Fbw7 would have a large impact on the clinics, but, so far, no potent compounds have been reported. Recently Bulatov et al. performed an exhaustive search within the PDB for crystal structures related to E3 ligases that can be very useful for drug discovery perspectives [13].

Another approach to target E3 ligases that has been underexploited is the search for allosteric molecules. This approach can result in dramatically different and unique pharmacological responses since E3 ubiquitin ligases have biochemically distinct druggable binding sites in their surfaces; molecules may turn the E3 ligase on as well as off. But on which basis can we start a project aimed at finding allosteric small molecules of the E3 ligases? Fragment-based screening is an established approach for interrogating target druggability. The main reason is that the 'fragments' are efficient probes to explore the chemical space at protein surfaces [14]. However, the bottleneck of fragment-based campaigns is obtaining $\mathrm{x}$-ray structures of the fragment-protein complex in order to follow a rational hit-to-lead optimization process.

Several computational tools have been developed in the last few years to identify new binding sites and predict their druggability [15]. This approach could be especially useful in the E3 ligases field, where sometimes the structure is available but it is not easy to envisage mol- 
ecules that can bind to the substrate binding site or the assemble PPIs. These computational tools can be classified in two main groups: grid-based mapping (GRID, SiteMap) and simulation-based mapping (MCSS, MDMix). In my opinion, the latter provides more trustworthy information. As an example of how they work, MDMix uses molecular dynamic simulations with aqueous/organic mixtures as solvation conditions [16]. It has been demonstrated by multiple experimental studies that proteins have the ability to bind small organic cosolvents in regions over the surface, and these regions correlate well with binding sites and important interactions for the drug binding affinity. Then, this information can be used for rational design or as a pharmacophore for virtual screening. Indeed, virtual screening has demonstrated to be useful to target E3 ligases, as recently shown by Chan et al. with the identification of an inhibitor of the tumor-promoting E3 ligase, Skp2 [17].

Screening of large libraries of compounds through functional assays (e.g., ubiquitation assays) could be another approach to target E3 ligases. However, several drawbacks can be considered in this case. In contrast to other families, a widely and general targeted library of compounds for E3 ligases is very difficult to prepare since the structural differences between the E3 ligases make this job unachievable. Targeted libraries for specific subfamilies of E3 ligases might be a better idea. In addition, the commonly used assays are typically low throughput and nonquantitative and need the recombinant expression and purification of the proteins involved in the E3 cascade. Additionally another in vitro screening based in fluorescence-based assays has been developed. This approach was used to identify an allosteric inhibitor of the yeast Cdc4 E3 ligase [18]. Finally, very few in-cell screening and generic methods have been described in order to find small molecules that target E3 ligases [19], however, I envisage an explosion of this kind of approaches in near future, which can yield small molecules already active in

\section{References}

1 Mullard A. 2015 FDA drug approvals. Nat. Rev. Drug Discov. 15(2), 73-76 (2016).

2 Cohen P, Tcherpakov M. Will the ubiquitin system furnish as many drug targets as protein kinases? Cell 143(5), 686-693 (2010).

3 Morreale FE, Walden H. SnapShot: types of ubiquitin ligases. Cell 165(1), 248-248.e1 (2016).

4 Huang X, Dixit VM. Drugging the undruggables: exploring the ubiquitin system for drug development. Cell Res. 26(4), 484-498 (2016).

5 Morrow JK, Lin HK, Sun SC, Zhang S. Targeting ubiquitination for cancer therapies. Fut. Med. Chem. 7(17), 2333-2350 (2015). a cell environment. Nevertheless, these approaches do not provide information about the binding site where the molecules bind.

In conclusion, there does not exist a general strategy to find molecules that target E3 ligases, and from my point of view each E3 ligase will have to be treated individually taken into account their biological, structural and functional characteristics. A balanced risk/gain assessment should be considered before starting a drug discovery project aiming to target E3 ligases and, crucially, the amount of knowledge for a given E3 ligase can foretell the chances of success. The difficulties inherent to this target family can be seen at the same time as advantages; there are opportunities to identify multiple chemical series with different mechanisms of action targeting a single E3 ligase that can provide more specific, more effective and less toxic drugs in the future.

\section{Acknowledgements}

The author thanks FJ Luque, D Muñoz-Torrero and X Barril for helpful discussions and manuscript revision. The author also thanks A Ciulli for introducing him to this extremely interesting field of E3 ligases.

\section{Financial \& competing interests disclosure}

The author is grateful to the organizations that have provided funding support, including the Secretaria d'Universitats i Recerca del Departament d'Economia i Coneixement de la Generalitat de Catalunya and the 7th European Framework Program-Marie Curie COFUND (contract 600385; Beatriu de Pinós Fellowship) and by the Spanish Ministerio de Economia y Competitividad (SAF2015-68749-R). The author has no other relevant affiliations or financial involvement with any organization or entity with a financial interest in or financial conflict with the subject matter or materials discussed in the manuscript apart from those disclosed.

No writing assistance was utilized in the production of this manuscript.

6 Berndsen CE, Wolberger C. New insights into ubiquitin E3 ligase mechanism. Nat. Struct. Mol. Biol. 21(4), 301-307 (2014).

7 Wells JA, McClendon CL. Reaching for high-hanging fruit in drug discovery at protein-protein interfaces. Nature 450 (7172), 1001-1009 (2007).

8 Skaar JR, Pagan JK, Pagano M. SCF ubiquitin ligasetargeted therapies. Nat. Rev. Drug Discov. 13(12), 889-903 (2014).

9 Galdeano C, Gadd MS, Soares P et al. Structure-guided design and optimization of small molecules targeting the protein-protein interaction between the von Hippel-Lindau (VHL) E3 ubiquitin ligase and the Hypoxia Inducible Factor (HIF) alpha subunit with In vitro nanomolar affinities. J. Med. Chem. 57(20), 8657-8663 (2014). 
10 Buckley DL, Van Molle I, Gareiss PC et al. Targeting the von Hippel-Lindau E3 ubiquitin ligase using small molecules to disrupt the VHL/HIF-1 $\alpha$ interaction. J. Am. Chem. Soc. 134(10), 4465-4468 (2012).

11 Bartesaghi A, Merk A, Banerjee $S$ et al. $2.2 \AA$ resolution cryo-EM structure of $\beta$-galactosidase in complex with a cellpermeant inhibitor. Science 348(6239), 1147-1151 (2015).

12 Hao B, Oehlmann S, Sowa ME, Harper JW, Pavletich NP. Structure of a Fbw7-Skp1-Cyclin E complex: multisitephosphorylated substrate recognition by SCF ubiquitin ligases. Mol. Cell 26(1), 131-143 (2007).

13 Bulatov E, Ciulli A. Targeting Cullin-RING E3 ubiquitin ligases for drug discovery: structure, assembly and smallmolecule modulation. Biochem. J. 467(3), 365-386 (2015).

14 Erlanson DA, Fesik SW, Hubbard RE, Jahnke W, Jhoti H. Twenty years on: the impact of fragments on drug discovery. Nat. Rev. Drug Discov. 15(9), 605-619 (2016).

15 Hussein HA, Geneix C, Petitjean M, Borrel A, Flatters D, Camproux A-C. Global vision of druggability issues: applications and perspectives. Drug Discov. Today doi:10.1016/j.drudis.2016.11.021 (2016) (Epub ahead of print).

16 Alvarez-Garcia D, Barril X. Molecular simulations with solvent competition quantify water displaceability and provide accurate interaction maps of protein binding sites. J. Med. Chem. 57(20), 8530-8539 (2014).

17 Chan C-H, Morrow JK, Li C-F et al. Pharmacological inactivation of Skp2 SCF ubiquitin ligase restricts cancer stem cell traits and cancer progression. Cell 154(3), 556-568 (2013).

18 Orlicky S, Tang X, Neduva V et al. An allosteric inhibitor of substrate recognition by the SCFCdc4 ubiquitin ligase. Nat. Biotechnol. 28(7), 733-737 (2010).

19 Maculins T, Carter N, Dorval T et al. A generic platform for cellular screening against ubiquitin ligases. Sci. Rep. 6, 18940 (2015). 